\title{
BRÈVES OBSERVATIONS SUR L'ARRÊT DE LA COUR INTERNATIONALE DE JUSTICE DU 17 MARS 2016, EN L'AFFAIRE DES VIOLATIONS ALLÉGUÉES DE DROITS SOUVERAINS ET D'ESPACES MARITIMES DANS LA MER DES CARAÏBES (NICARAGUA C. COLOMBIE). REVUE GÉNÉRALE DE DROIT INTERNATIONAL PUBLIC, 2016-2.
}

Pierre-François LAVAL

Laffaire des Violations alléguées de droits souverains et d'espaces maritimes dans la mer des Caraïbes promettait d'être historique (voir pour une analyse des circonstances de l'affaire avant le prononcé de l'arrêt du 17 mars 2016, G. Vidigal, "Compliance Adjudication at the ICJ - The Alleged Violations Case ", EJIL: Talk !, 26 janvier 2016). Voilà plus de soixante ans que la Cour internationale de Justice n'avait pas été saisie d'une demande mettant directement en cause l'exécution de l'un de ses arrêts! L'initiative prise en ce sens par le Nicaragua trouve son origine dans les vives réactions suscitées par l'arrêt du 19 novembre 2012 (Différend territorial et maritime (Nicaragua c. Colombie), CIJ Rec. 2012, pp. 624-720), que les termes utilisés par le juge Abraham dans son opinion individuelle laissaient d'ailleurs parfaitement présagerı. Rappelons que dix jours à peine après le prononcé de l'arrêt, le Président Juan Manuel Santos décidait de retirer la Colombie du Pacte de Bogota de 1948 afin de ne plus s'exposer aux jugements de la Cour de La Haye. Le 10 septembre de l'année suivante, il déclarait «non applicable » l'arrêt de la Cour sur le fondement de la Constitution colombienne qui impose la conclusion d'un traité pour toute modification des frontières. Ces prises de position s'accompagneront d'une série d'évènements que la requête nicaraguayenne, déposée le 26 novembre 2013, présentait tout à la fois comme une violation des droits souverains et des espaces maritimes que la Cour lui avait reconnus dans son arrêt du 19 novembre 2012, et comme un manquement à lobligation de s'abstenir de recourir à la menace ou à l'emploi de la force.

Faisant suite à celles introduites deux mois plus tôt au titre de la délimitation du plateau continental au-delà des 200 milles marins (arrêt du 17 mars 2016, Question de la délimitation du plateau continental entre le Nicaragua et la Colombie au-delà de 200 milles marins de la côte nicaraguayenne, dont le commentaire de N. Aloupi suit la présente note), les demandes du Nicaragua invitaient ainsi la Cour à livrer ses vues sur une question qu'elle n'avait plus directement

\footnotetext{
Professeur de droit public à l'Université d'Orléans

1 Opinion individuelle jointe à l'arrêt du 19 novembre 2012, CIJ Rec. 2012, p. 739, \$35: « [j]e comprends que la Cour veuille donner à tous ceux qui l'observent, et d'abord aux Etats, le sentiment qu'elle ne procède pas de manière arbitraire pour parvenir à une solution équitable, mais qu'elle met en œuvre des techniques éprouvées et constantes. Et il est parfaitement vrai qu'il n'y a aucun arbitraire dans la démarche de la Cour, mais seulement la recherche scrupuleuse de la meilleure solution. Il est cependant des affaires qui se présentent en des termes tellement spécifiques qu'il est, à tout prendre, préférable de reconnaître que la Cour doit s'écarter de sa technique habituelle, en expliquant pourquoi, plutôt que de sacrifier la clarté et l'intelligibilité à l'apparence d'une illusoire continuité ».
} 
abordée depuis l'affaire Haya de la Torre de 1951. A l'occasion de cette dernière affaire, la Cour avait jugé irrecevable la demande adressée par la Colombie en vue dobtenir des précisions sur les modalités d'exécution d'un arrêt rendu quelques mois plus tôt. Rappelant la liberté de choix bénéficiant en la matière aux Etats, elle avait estimé qu'il ne relevait pas de sa fonction judiciaire de trancher entre les différentes voies par lesquelles ses arrêts pourraient être exécutés, à plus forte raison lorsque de tels choix sont « conditionnés par des éléments de fait et par des possibilités que, dans une très large mesure, les Parties sont seules en situation d'apprécier " (arrêt du 31 juin 1951, CIJ Rec. 1951, spéc. pp. 78-79). La problématique de l'exécution était, dès lors, envisagée comme un phénomène essentiellement politique, susceptible d'échapper à l'emprise du droit (voir en ce sens Sh. Rosenne, «L'exécution et la mise en vigueur des décisions de la Cour internationale de Justice, RGDIP, 1953, pp. 532-583, spéc. p. 538). Certains en avaient toutefois imprudemment conclu que la Cour refuserait d'examiner toute affaire touchant, de près ou de loin, à l'obligation d'exécuter ses arrêts, au point que des requêtes clairement liées à un problème d'exécution furent travesties en demandes en interprétation dans l'affaire Avena, puis dans celle relative au Temple de Préah Vihéar. La Cour pu alors, en ces différentes occasions, réitérer son refus de prêter assistance à l'exécution de ses décisions (voir Demande en interprétation de l'arrêt du 31 mars 2004 en l'affaire Avena et autres ressortissants mexicains (Mexique c. EtatsUnis), arrêt du 19 janvier 2009, CIJ Rec. 2009, p. 20, \$56). La cinquième exception préliminaire soulevée par la Colombie aura toutefois permis à la Cour, dans son arrêt rendu le 17 mars dernier, de distinguer l'hypothèse de l'assistance à l'exécution, de la compétence pour trancher un différend ayant pour objet l'obligation d'exécuter un précédent jugement (voir sur ce point C. Santulli, Droit du contentieux international, $2^{\text {ème }}$ édition, 2015, pp. 517-518).

L'argumentaire colombien visait en effet à démontrer que le Nicaragua sollicitait une nouvelle décision de la Cour qui permettrait d'assurer l'exécution de l'arrêt du 19 novembre 2012. Une telle demande avait toutefois, toujours aux dires des autorités colombiennes, été effectuée en méconnaissance de la stricte séparation des pouvoirs établie par la Charte des Nations Unies et le Statut de la Cour, aux termes de laquelle « la Cour a pour tâche de régler les différends tandis qu'il appartient au Conseil de Sécurité de veiller à l'exécution des arrêts une fois quelle a statué, conformément au paragraphe 2 de l’article 94 de la Charte » (\$105). La saisine de la Cour devait, en d'autres termes, s'analyser en une stratégie de contournement du Conseil de Sécurité, pourtant seul compétent en matière d'exécution des arrêts de la Cour (voir ici la déclaration du juge Bhandari acquis à cette thèse). La haute juridiction n’adhère toutefois pas à cette analyse. Tout en reconnaissant que l'arrêt de 2012 est incontestablement au cœur de la présente affaire, elle estime que lobjet de la requête déposée par le Nicaragua est d’obtenir le constat la violation de ses droits par les autorités colombiennes, ainsi que la réparation intégrale du préjudice dont celles-ci sont à l'origine, avant de sobrement conclure que « le Nicaragua ne cherche pas à faire exécuter l'arrêt de 2012 en tant que tel » ( $\$ 109$ de l’arrêt). La formule, laconique, établit ainsi de façon tout à fait inédite la compétence de la Cour pour connaître d'un différend relatif à l'exécution de l'une de ses précédentes décisions. La même solution sera retenue dans le second arrêt Question de la délimitation du plateau continental, aux dépens d'un argumentaire colombien légèrement modifié et articulé autour d'une prétendue tentative « d'appel » ou de « révision » de l'arrêt de 2012 (Question de la délimitation du plateau continental..., $\$ \$ 89-90$ ). Le raisonnement de la Cour, qui aurait sans doute gagné à être plus amplement étayé, semble ainsi reposer sur l’altérité des situations litigieuses mises en cause : la non-exécution d'un jugement de la Cour constitue un fait internationalement illicite auquel sont assorties des conséquences juridiques indépendantes de celles constatées par ledit jugement, et susceptibles de faire l’objet d'un différend lui-même pleinement autonome. Régler le litige sur l'exécution du premier jugement ne revient donc pas 
procéder à son exécution même, mais à statuer sur de nouvelles prétentions et, le cas échéant, à établir de nouveaux droits.

Le traitement des autres exceptions préliminaires colombiennes a, pour sa part, fourni d'utiles indications sur les conditions dans lesquelles un différend de cette nature pourrait être valablement soumis à la Cour. La principale confirmation concerne la nécessité de saisir cette dernière sur le fondement d'une base de compétence opposable, à cette même date, à l'Etat défendeur. En cela, l'action introduite par le Nicaragua peut être aisément distinguée d'une demande en interprétation ou en révision pour lesquelles les articles 60 et 61 du Statut de la Cour n'imposent pas l'existence d'une base de compétence autre que celle ayant fondé le premier jugement. Larrêt du 17 mars ne fait toutefois qu'effleurer cette importante distinction entre les différentes actions. Ayant conclu que le Pacte de Bogota liait encore les deux parties au jour du dépôt de la requête nicaraguayenne, la Cour n’a effectivement pas jugé utile d'évaluer la thèse, subsidiairement défendue par la Colombie, d'un prétendu " pouvoir inhérent » qui l'autoriserait, en tout état de cause, à se prononcer sur les « mesures requises par ses arrêts » (Quatrième exception préliminaire, $\$ \$$ 102-104 de l'arrêt ; voir également l'analyse détaillée du juge Cançado Trindade dans son opinion séparée jointe à l'arrêt, spéc. $\$ \$ 67-75$. Sous des traits quelque peu modifiés, la même thèse sera développée au titre d'une prétendue " compétence continue » dans l'affaire Question de la délimitation du plateau continental, deuxième exception préliminaire, $\$ \$ 91-94$ ).

Lexception préliminaire ayant le plus retenu l'attention de la Cour au vu des longs développements qu'elle y consacre, et qui sont repris verbatim dans l'affaire relative à la Question de la délimitation du plateau continental, concernait les effets de la dénonciation du Pacte par la Colombie ( $\$ \$ 20-48)$. La Cour débute son analyse par un rappel des règles régissant sa compétence ratione temporis, notamment celle qui lui commande d'apprécier sa compétence au jour du dépôt de la requête $(\$ 33)$. La justification donnée à ce principe dans le droit du contentieux international tient à la conséquence que l'on peut y attacher : à supposer que la compétence soit établie à la date de la saisine, aucun évènement n'est plus susceptible de la remettre en cause d'ici la date du jugement (voir notamment Nottebohm (Liechtenstein c. Guatemala), arrêt sur les exceptions préliminaires du 18 novembre 1953, CIJ Rec. 1953, pp. 111-125, spéc. p. 123). Lapplication de cette règle ne pouvait toutefois suffire in casu à faire justice de l'exception temporelle soulevée par les autorités colombiennes. Dans leur avis de dénonciation, celles-ci avaient effectivement pris le soin d'indiquer que la dénonciation prendrait effet « à compter du jour [même] à l'égard des procédures introduites postérieurement [à l']avis, conformément au second alinéa de l'article LVI [du Pacte de Bogota] ». Elles avaient, ce disant, opéré une interprétation a contrario de cette dernière disposition au terme de laquelle « la dénonciation [du Pacte] n'aura aucun effet sur les procédures en cours entamées avant la transmission de l'avis en question ». Devant la Cour, la partie colombienne estimait qu'il découlait " naturellement » d'un tel libellé que sa dénonciation avait bel et bien un effet à l'égard des procédures entamées après transmission de l'avis, et ainsi à l'égard de celle engagée par le Nicaragua en la présente affaire. Il reste que cette disposition de l'article LVI est précédée d'un premier alinéa prévoyant que la dénonciation du Pacte peut intervenir « moyennant un préavis d'un an ; passé ce délai, [le Pacte] cessera de produire ses effets par rapport à la partie qui l’a dénoncé ». La requête nicaraguayenne ayant été soumise, dans la présente affaire, après la transmission de l'avis, mais avant l'expiration du préavis d'un an, la Cour était ainsi tenue d'évaluer la pertinence de l'interprétation colombienne. La principale difficulté consistait à donner sa juste signification à la formulation pour le moins malheureuse du second alinéa, tout en l'accordant avec la disposition du premier alinéa. On conviendra effectivement que la précaution prise par les rédacteurs du Pacte de préciser que la dénonciation est dépourvue d’effets sur les procédures déjà entamées, apparaît totalement 
superflue. Un tel résultat est déjà garanti par les dispositions du premier alinéa de l'article LVI qui, comme le rappelle la Cour, seul détermine les effets ratione temporis de la dénonciation en prévoyant que l'extinction du Pacte n'intervient qu'au terme d'un délai d'un an à compter de la décision de dénoncer. Le caractère superfétatoire de la clause suffit-il alors à valider la lecture a contrario avancée par les autorités colombiennes ? Nullement, si l'on s'en tient au raisonnement unanimement suivi par les membres de la Cour. La technique de l'interprétation a contrario a, certes, déjà été employée par la Cour au cours de précédentes affaires, notamment celle relative au Différend territorial et maritime (Nicaragua c. Colombie, requête du Honduras à fin d'intervention, arrêt du 4 mai 2011, CIJ Rec. pp. 420-445, spéc. p. 432, \$29). La Cour estime toutefois ne devoir y recourir que si son emploi « se justifie à la lumière du libellé de l'ensemble des dispositions pertinentes, de leur contexte ainsi que de l'objet et du but du traité » $(\$ 37)$. Or, dans le cas d’espèce, l’interprétation avancée par la Colombie ne résiste pas à la prise en considération de ces différents éléments. Exclure la possibilité de recourir aux procédures dès la transmission de l'avis de dénonciation reviendrait à priver le Pacte de son principal objet - celui d'imposer une obligation générale de régler les différends par des moyens pacifiques pour toute la période où ses effets sont maintenus par le libellé expresse du premier alinéa de l'article LVI. La Cour rappelle par ailleurs que l'opération d'interprétation d'un traité suppose de " veiller à ce qu'aucune de ses dispositions ne soit privée de portée ou d'effet ", mais convient dans le même temps « qu'il arrive (...) que les parties à un traité adoptent une disposition afin d'éviter tout doute, même si celle-ci n’est pas strictement nécessaire » $(\$ 43)$. A ses yeux, la seule interprétation respectueuse des effets du premier alinéa suppose de considérer que les parties au Pacte ont inclus la disposition du second alinéa par surcroît de prudence, dans le seul but de confirmer que « les procédures introduites avant la transmission de l'avis de dénonciation peuvent en tout état de cause se poursuivre» $(\$ 39)$. Au terme de son analyse, la Cour estime donc sa compétence établie ratione temporis, la Colombie étant toujours liée par les dispositions du Pacte de Bogota à la date à laquelle le Nicaragua avait introduit l'instance.

Les deux dernières exceptions préliminaires étaient, pour leur part, liées à d'autres conditions posées ou simplement rappelées par le Pacte de Bogota au sujet de l'exercice par la Cour de sa compétence contentieuse. La première devait conduire cette dernière à retracer la séquence d'évènements seetant succédé à compter de son précédent arrêt, en vue de déterminer l'existence d'un différend entre les parties en rapport avec les demandes du Nicaragua au jour de leur dépôt. La Cour estime qu'un litige sétait bien constitué au titre de la première demande relative à la violation des espaces maritimes. L'antagonisme avéré des thèses développées par les deux parties au sujet de leurs droits respectifs dans les espaces maritimes visés par l'arrêt de 2012 ( $\$ \$$ 67-70) conduit, selon la Cour, à écarter la thèse selon laquelle la requête aurait constitué une «totale surprise » pour les autorités colombiennes (voir, a contrario, l'opinion dissidente du juge ad hoc Caron, spéc. $\$ \$ 28-60)$. Le fait même que ces dernières aient entamé un dialogue avec la partie adverse, en vue de conclure un traité international permettant de donner plein effet audit arrêt n'est pas de nature à remettre en cause cette conclusion, de même que l'absence de protestation diplomatique officielle qui, aux dires la Cour, n'est pas une " condition nécessaire » $(\$ 72)$ pour établir l'existence du conflit. Le litige relatif aux espaces maritimes était toutefois le seul qui, de l'avis de la Cour, opposait les parties au jour du dépôt de la requête. Rien ne laissait effectivement entendre que le Nicaragua ait, à un quelconque moment avant la saisine, indiqué à la Colombie le manquement à ses obligations en matière de recours à la menace ou à l'emploi de la force $(\$ 76)$. L'exception préliminaire colombienne est ainsi accueillie au seul titre de la seconde demande nicaraguayenne. En dernière analyse, la Cour était invitée à se pencher sur le respect du préalable juridictionnel de l'article II du Pacte qui impose aux Etats 
parties, avant tout recours au règlement judiciaire, de chercher à résoudre leur litige au moyen de " procédures pacifiques régionales ». Suivant une méthodologie déjà éprouvée (voir Actions armées frontalières et transfrontalières (Nicaragua c. Honduras), arrêt du 20 décembre 1988 sur la compétence et la recevabilité, CIJ Rec. 1988, pp. 69-107, spéc. pp. 94-95, \$\$ 65-66), la Cour estime devoir s'en tenir aux éléments produits par les parties en vue d'évaluer les possibilités d’un règlement négocié ( $\$ \$ 92-95)$. Examinant sous cet aspect le dialogue engagé par les chefs d'Etat depuis 2012, la Cour relève qu'il s'est limité aux modalités d'exécution de l'arrêt de la Cour sans jamais intégrer la controverse naissante sur le non-respect des droits reconnus au Nicaragua dans ses espaces maritimes. Aucune partie ne pouvant soutenir de manière plausible que le différend était susceptible de trouver une issue suivant les voies diplomatiques ordinaires, l'exception préliminaire est rejetée. 
